\title{
September 2018 Arizona Thoracic Society Notes
}

The September 2018 Arizona Thoracic Society meeting was held on Wednesday, September 26, 2018 at the HonorHealth Rehabilitation Hospital beginning at 6:30 PM. This was a dinner meeting with case presentations. There were representatives in attendance from the pulmonary, critical care, sleep, and radiology communities.

At the beginning of the meeting attendance was again discussed.

There were 3 case presentations:

1. Dr. Gerry Schwartzberg presented a case of a woman in her 70's with diffuse cystic lung disease. She had a monoclonal gammopathy of undetermined significance due to light chain disease and the possibility of amyloidosis causing cystic lung disease was discussed.

2. Dr. Lewis Wesselius presented a 28-year-old man from Tennessee with enlarging lung nodules who had been treated for presumed histoplasmosis, but was having hemoptysis and clinically worsening. Lung biopsy demonstrated metastatic angiosarcoma.

3. Dr. Wesselius also presented a 44-year-old woman with diabetes and cavitary pulmonary coccidioidomycosis which involved both left upper lobe and left lower lobe which had progressed despite $400 \mathrm{mg} /$ day of fluconazole who had been referred in 2012 for possible thoracotomy. Surgery was deferred since it would have required a left pneumonectomy. The fluconazole dose was increased, and cavity resolved gradually over 6 years. This led to discussion of indications for surgery in cavitary coccidioidomycosis.

The meeting was adjourned about 8 PM. The next meeting will be on November 28 at 6:30 PM at HonorHealth Rehabilitation Hospital.

Lewis J. Wesselius, MD

President, Arizona Thoracic Society 